\title{
EDITORIAL
}

\section{Fatigued muscles in COPD but no finishing line in sight}

\author{
H.R. Gosker and A.M.W.J. Schols
}

D ecreased exercise capacity is a prominent symptom leading to progressive disablement in chronic obstructive pulmonary disease (COPD). Exercise capacity is frequently measured by means of incremental cycle ergometry. Although a well-established test, studies are ongoing to improve test design, test variables and data interpretation. For example, a high-intensity constant-work rate cycle ergometer test appears to be more informative than an incremental test to evaluate the outcome of rehabilitative interventions. At present, however, incremental cycle ergometry is considered the gold standard for evaluating the pathophysiology of exercise impairment in COPD [1]. Besides decreased ventilatory function, skeletal muscle dysfunction has been identified as an important determinant of exercise limitation in moderate-to-severe COPD. Impaired muscle function can manifest itself as the loss of strength and/or as reduced endurance (i.e. fatigue). Both are obviously involved in COPD but their relative contribution to exercise intolerance in individual patients is as yet unclear. In healthy subjects as well as in COPD patients, muscle strength depends largely on muscle mass or fat-free mass; fat-free mass has indeed been found to be a strong predictor of exercise capacity [2]. However, the relationship between fat-free mass (i.e. muscle strength) and exercise capacity was much weaker in COPD patients as compared with healthy controls, which may point to a more prominent role for muscle fatigue in COPD.

But how to proceed? First of all, there is an urgent need for adequate muscle function tests and reference values. The determination of muscle fatigue, however, is very complicated since there is no uniform definition of muscle fatigue and available tests are not comparable.

\section{FUNCTIONAL ASSESSMENT OF MUSCLE FATIGUE}

Muscle fatigue can be defined as the reversible loss of muscle force due to work over time. Several studies have implemented this concept to evaluate leg muscle fatigue in COPD. Some measured the decrease in maximal force over a sustained isometric contraction [3] or in a series of isokinetic maximal voluntary contractions [2]. These tests are powerful (maximal contractions) and of rather short duration $(<1 \mathrm{~m})$. Exercise protocols of longer duration $(\sim 2-15 \mathrm{~min})$ have also been

Dept of Respiratory Medicine, NUTRIM School for Nutrition, Toxicology and Metabolism, Maastricht University, Maastricht, The Netherlands.

STATEMENT OF INTEREST: None declared.

CORRESPONDENCE: A.M.W.J. Schols, Dept of Respiratory Medicine, NUTRIM School for Nutrition, Toxicology and Metabolism, Maastricht University, P0 Box 6161, 6200 MD, Maastricht, The Netherlands. Fax: 31 433875051. E-mail: a.schols@pul.unimaas.nl applied: the duration and work of a series of dynamic submaximal voluntary contractions until exhaustion [4]; the drop in involuntary muscle force measured by magnetic stimulation pre- and post-whole body [5, 6] or single leg [7] fatiguing tasks; electromyogram analysis during cycling exercise $[6,8]$; and the fall in maximal twitch force during repetitive magnetic stimulation [9]. Subjective leg fatigue has also been evaluated, mostly by comparing pre- and postexercise Borg or visual analogue scale scores $[6,10]$. The large diversity in the nature of these exercises, such as variations in duration, intensity and stimulation frequency, is likely to result in different kinds of muscle fatigue. For example, short extensive exercise muscle contractions depend highly on the fast-twitch muscle fibres, the phosphocreatine buffer and glycolytic metabolism, whereas prolonged exercise depends more on slow-twitch fibres and oxidative metabolism.

\section{METABOLIC ASSESSMENT OF MUSCLE FATIGUE}

Reduced muscle endurance in COPD occurs independently of the loss of muscle mass; its origin must therefore be sought in the intrinsic muscular alterations, such as reduced fibre type I proportion and decreased oxidative enzyme activities [2]. In vivo studies using noninvasive ${ }^{31} \mathrm{P}$-nuclear magnetic resonance revealed that muscular levels of high-energy phosphates (e.g. adenosine triphosphate (ATP), phospocreatine and reduced nicotinamide adenine dinucleotide) are reduced at rest and that their rephosphorylation during and after physical exercise is slower in COPD patients as compared with healthy control subjects, indicative of impaired oxidative energy metabolism. As oxidative phosphorylation of adenosine diphosphate (ADP) to produce ATP fails to meet the energy demand, the muscle cell switches to anaerobic metabolism resulting in the formation of lactate. Elevated blood lactate during exercise is frequently used to monitor muscle fatigue during cycle ergometry, and in COPD an early onset of the lactate threshold has indeed been associated with reduced muscle oxidative capacity [11].

Another marker to monitor the onset of muscle fatigue is blood ammonia, which normally closely follows the lactate response during exercise. As muscle ATP consumption exceeds ATP supply, the ATP / ADP ratio drops, impairing the functions of the primary ATP consumers in the muscle (myosin adenosine triphosphatase $(70 \%)$ and sarcoplasmic reticulum ATPase $(25 \%))$, leading to the loss of muscle contractility (i.e. fatigue). To maintain the ATP/ADP ratio the enzyme adenylate kinase transfers an energy-rich phosphate group from one ADP to another ADP, resulting in one ATP and one adenosine monophosphate (AMP) molecule. AMP is subsequently degraded to inosine monophosphate (IMP) and ammonia by the enzyme AMP deaminase [12]. CALVERT et al. [13] were the first to study ammonia levels during cycle ergometry in COPD. 
In this study presented in the current issue of the European Respiratory Journal, they found that, compared with healthy controls, $\sim 60 \%$ of the COPD patients exhibited a rise in blood ammonia associated with a rise in intramuscular IMP levels during cycle exercise, despite a lower absolute work load. This implies that the relative ammonia production is higher in these patients, which in turn suggests that enhanced AMP degradation as a consequence of insufficient ATP production to meet energy demands occurred. This finding fits with the reduced muscle oxidative phenotype of COPD patients. In addition, hydrogen peroxide is formed by the enzyme xanthine oxidase in the further degradation of IMP into uric acid, which may lead to loss of muscle contractility and thus augment muscle fatigue [14].

Remarkably, in the study by CALVERT et al. [13], a clear ammonia response was absent in nine out of $24(\sim 40 \%)$ COPD patients during incremental cycling. As AMP deaminase activity is predominant in glycolytic type II fibres, CALVERT et al. [13] suggested that the I to II fibre type shift, which has consistently been reported for COPD, might not be present in this particular subgroup of patients. However, this is unlikely as it would imply that muscle fibre type distribution resembles that of the healthy controls who did show a rise in ammonia. An alternative, although very speculative, explanation could be a bias introduced through use of $\beta_{2}$-adrenoreceptor agonists prior to the exercise test, since it has been shown that acute administration of these drugs decreases blood ammonia levels during exercise in healthy subjects [15]. A more plausible explanation, also provided by CALVERT et al. [13], is that ATP supply met ATP demand during exercise in this subgroup. It has indeed been shown that for cycling, $\sim 40 \%$ of the COPD patients specified dyspnoea as a limiting factor [16], indicating that the leg muscles may not yet have reached their fatigue threshold. However, the fact that blood lactate increased despite the absence of the rise in blood ammonia in these patients suggests otherwise. Another explanation for the absence of the ammonia response could relate to an abnormal protein metabolic response after exercise. ENGELEN et al. [17] reported that, in contrast to healthy control subjects, protein turnover did not show the expected increase during cycle exercise in a subgroup of COPD patients.

For a better understanding of muscle fatigue in chronic obstructive pulmonary disease it is crucial to validate the functional and metabolic indices of fatigue against each other. For example, muscle fatigue was found to be associated with reduced muscle oxidative capacity and slow-fibre type proportions in the repetitive magnetic stimulation test mentioned previously [9]. It would be interesting to also measure lactate and/or ammonia kinetics during this test, as it would tell us whether this is a similar fatigue to that which occurs during the cycle ergometry tests. With this approach the nature and relative contribution of muscle fatigue to exercise intolerance in chronic obstructive pulmonary disease can be identified. Only then will the finishing line for fatigued muscles be in sight and the next challenge can then be undertaken to improve muscle performance: individually tailored intervention strategies.

\section{REFERENCES}

1 Palange $\mathrm{P}$, Ward SA, Carlsen $\mathrm{KH}$, et al. Recommendations on the use of exercise testing in clinical practice. Eur Respir J 2007; 29: 185-209.
2 Gosker HR, Lencer NHMK, Franssen FME, Wouters EFM, van der Vusse GJ, Schols AMWJ. Striking similarities in systemic factors contributing to decreased exercise capacity in patients with severe chronic heart failure or COPD. Chest 2003; 123: 1416-1424.

3 Zattara-Hartmann MC, Badier M, Guillot C, Tomei C, Jammes Y. Maximal force and endurance to fatigue of respiratory and skeletal muscles in chronic hypoxemic patients: the effects of oxygen breathing. Muscle Nerve 1995; 18: 495-502.

4 Serres I, Varray A, Vallet G, Micallef JP, Préfaut C. Improved skeletal muscle performance after individualized exercise training in patients with chronic obstructive pulmonary disease. J Cardiopulm Rehabil 1997; 17: 232-238.

5 Mador MJ, Kufel TJ, Pineda LA. Quadriceps fatigue after cycle exercise in patients with chronic obstructive pulmonary disease. Am J Respir Crit Care Med 2000; 161: 447-453.

6 Saey D, Cote $\mathrm{CH}$, Mador MJ, et al. Assessment of muscle fatigue during exercise in chronic obstructive pulmonary disease. Muscle Nerve 2006; 34: 62-71.

7 Mador MJ, Deniz O, Aggarwal A, Kufel TJ. Quadriceps fatigability after single muscle exercise in patients with chronic obstructive pulmonary disease. Am J Respir Crit Care Med 2003; 168: 102-108.

8 Gosselin N, Matecki S, Poulain M, et al. Electrophysiologic changes during exercise testing in patients with chronic obstructive pulmonary disease. Muscle Nerve 2003; 27: 170-179.

9 Swallow EB, Gosker HR, Ward KA, et al. A novel technique for nonvolitional assessment of quadriceps muscle endurance in humans. J Appl Physiol 2007; 103: 739-746.

10 Stendardi L, Grazzini M, Gigliotti F, Lotti P, Scano G. Dyspnea and leg effort during exercise. Respir Med 2005; 99: 933-942.

11 Maltais F, Simard AA, Simard C, Jobin J, Desgagnes P, LeBlanc P. Oxidative capacity of the skeletal muscle and lactic acid kinetics during exercise in normal subjects and in patients with COPD. Am J Respir Crit Care Med 1996; 153: 288-293.

12 Hancock CR, Brault JJ, Terjung RL. Protecting the cellular energy state during contractions: role of AMP deaminase. J Physiol Pharmacol 2006; 57: Suppl. 10, 17-29.

13 Calvert LD, Singh SJ, Greenhaff PL, Morgan MD, Steiner $\mathrm{MC}$. The plasma ammonia response to cycle exercise in COPD. Eur Respir J 2008; 31: 751-758.

14 Reid MB, Stokic DS, Koch SM, Khawli FA, Leis AA. Nacetylcysteine inhibits muscle fatigue in humans. J Clin Invest 1994; 94: 2468-2474.

15 Matthys D, Calders P, Pannier JL. Inhaled salbutamol decreases blood ammonia levels during exercise in normal subjects. Eur J Appl Physiol Occup Physiol 1998; 79: 110-113.

16 Pepin V, Saey D, Whittom F, LeBlanc P, Maltais F. Walking versus cycling: sensitivity to bronchodilation in chronic obstructive pulmonary disease. Am J Respir Crit Care Med 2005; 172: 1517-1522.

17 Engelen MP, Deutz NE, Mostert R, Wouters EF, Schols AM. Response of whole-body protein and urea turnover to exercise differs between patients with chronic obstructive pulmonary disease with and without emphysema. Am J Clin Nutr 2003; 77: 868-874. 